\title{
The Adhesive Response of Regolith to Low-Energy Disturbances in Microgravity
}

Stephanie Jarmak ${ }^{1}$, Joshua Colwell ${ }^{2}$, Adrienne Dove², Julie Brisset ${ }^{2}$

${ }^{1}$ Southwest Research Institute, 6220 Cluebra Rd, San Antonio TX 78238; '2University of Central Florida, 4000 Central Florida Blvd, Orlando FL 32816

Abstract

Small, airless bodies are covered by a layer of regolith composed of particles ranging from $\mu$ m-size dust to $\mathrm{cm}$-size pebbles that evolve under conditions very different than those on Earth. Flight-based microgravity experiments investigating lowvelocity collisions of $\mathrm{cm}$-size projectiles into regolith have revealed that certain impact events result in a mass transfer from the target regolith onto the surface of the projectile. The key parameters that produce these events need to be characterized to understand the mechanical behavior of granular media, which is composed of the surfaces of small bodies. We carried out flight and ground-based research campaigns designed to investigate these mass transfer events. The goals of our experimental campaigns were (1) to identify projectile energy thresholds that influence mass transfer outcomes in low-energy collision events between $\mathrm{cm}$-size projectiles and $\mu \mathrm{m}$-size regolith, (2) to determine whether these mass transfer events required a microgravity environment to be observed, and (3) to determine whether the rebound portion of these collision events could be replicated in a laboratory drop tower environment. We found that (1) mass transfer events occurred for projectile rebound accelerations $<7.8 \mathrm{~m} / \mathrm{s}^{2}$ and we were unable to identify a corresponding impact velocity threshold, (2) mass transfer events require a microgravity environment, and (3) ourdrop tower experiments were able to produce mass transfer events. However, drop tower experiments do not exactly reproduce the free-particle impacts and rebound of the long-duration microgravity experiments and yielded systematically lower amounts of the overall mass transferred.

Keywords

Asteroid $\bullet$ Adhesion $\bullet$ Impact $\bullet$ Microgravity $・$ Regolith

\section{INTRODUCTION}

Small planetary bodies, a class of solar system objects smaller than dwarf planets that are unlikely to have undergone differentiation, are considered tracers of the early state of the solar system and are therefore important to understand the origin and evolution of planetary systems. These bodies are represented by the targets of two current sample return missions [Hayabusa-2 (Smith, 2019) and OSIRIS-Rex (Lauretta et al., 2019)] along with several planned exploration missions, and these bodies also host valuable resources for future In-Situ Resource Utilization (ISRU) enterprises (Binzel, 2014).

Particles in the micrometer-centimeter size regime accrete through low-velocity ( $10 \mathrm{~s}$ of $\mathrm{cm} / \mathrm{s}$ ) collisions into meter- to kilometer-sized bodies within planetary ring systems and form a layer of regolith on small, airless bodies (Gundlach \& Blum, 2013). This regolith evolves under conditions very different from those on Earth as a result of significantly reduced gravity and pressure conditions. Additionally, spacecraft associated with planned and recent exploration missions interact with this regolith at speeds under $1 \mathrm{~m} / \mathrm{s}$ to sample the surface. Therefore, characterizing the response of regolith to low-energy impacts in a microgravity environment is critical to our understanding of the processes that lead to the formation of these objects and our ability to develop safe operating procedures on their surfaces for future exploration and resource utilization missions.

We have carried out several flight-based microgravity experiments in a vacuum designed to investigate low-velocity impacts of $\mathrm{cm}$-scale projectiles into simulated planetary regolith to contribute to a better understanding of the response of the surfaces of small, airless bodies to low-energy disturbances. These experiments include the most recent experiments in the COLLIDE (Collisions Into Dust Experiment) and PRIME (Physics of Regolith Impacts in Microgravity Experiment) programs carried out in suborbital flight (COLLIDE-3), and on parabolic airplane flights (PRIME-3, PRIME-4). From these experiments, we observed that certain impact events occurring at speeds less than $53 \mathrm{~cm} / \mathrm{s}$ resulted in mass transfer, in which material from the target regolith is retained on the projectile after the rebound. Figures 1 and 2 show examples of mass transfer events for

${ }^{\dagger}$ Corresponding author: Stephanie Jarmak

E-mail: sjarmak@swri.org 


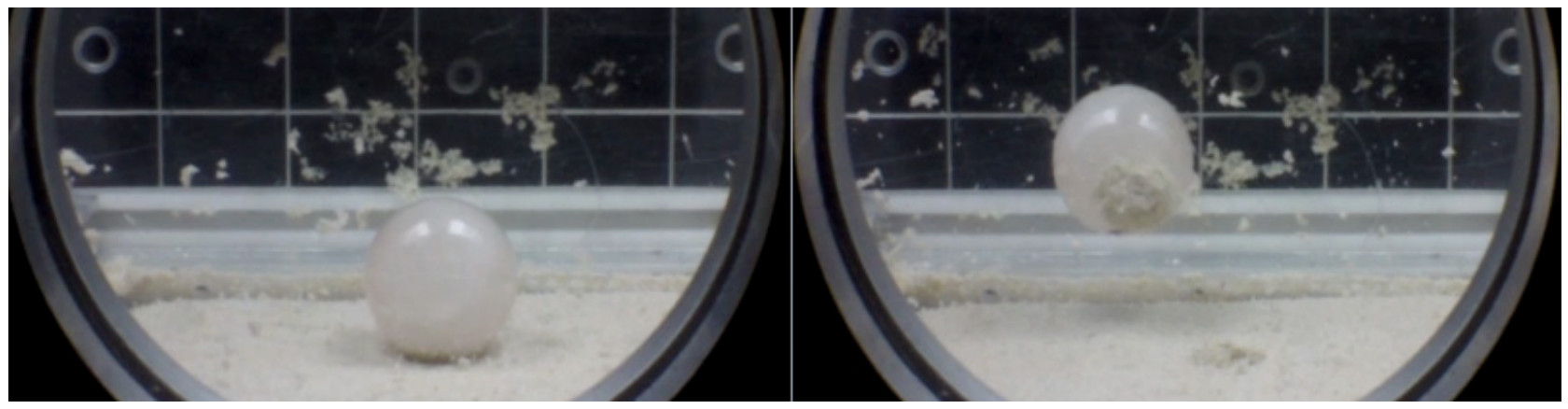

Figure 1. Still frames of a 10-g quartz impactor (left) contacting quartz sand at an impact speed of $23 \mathrm{~cm} / \mathrm{s}$ and (right) rebounding with observable mass transfer.

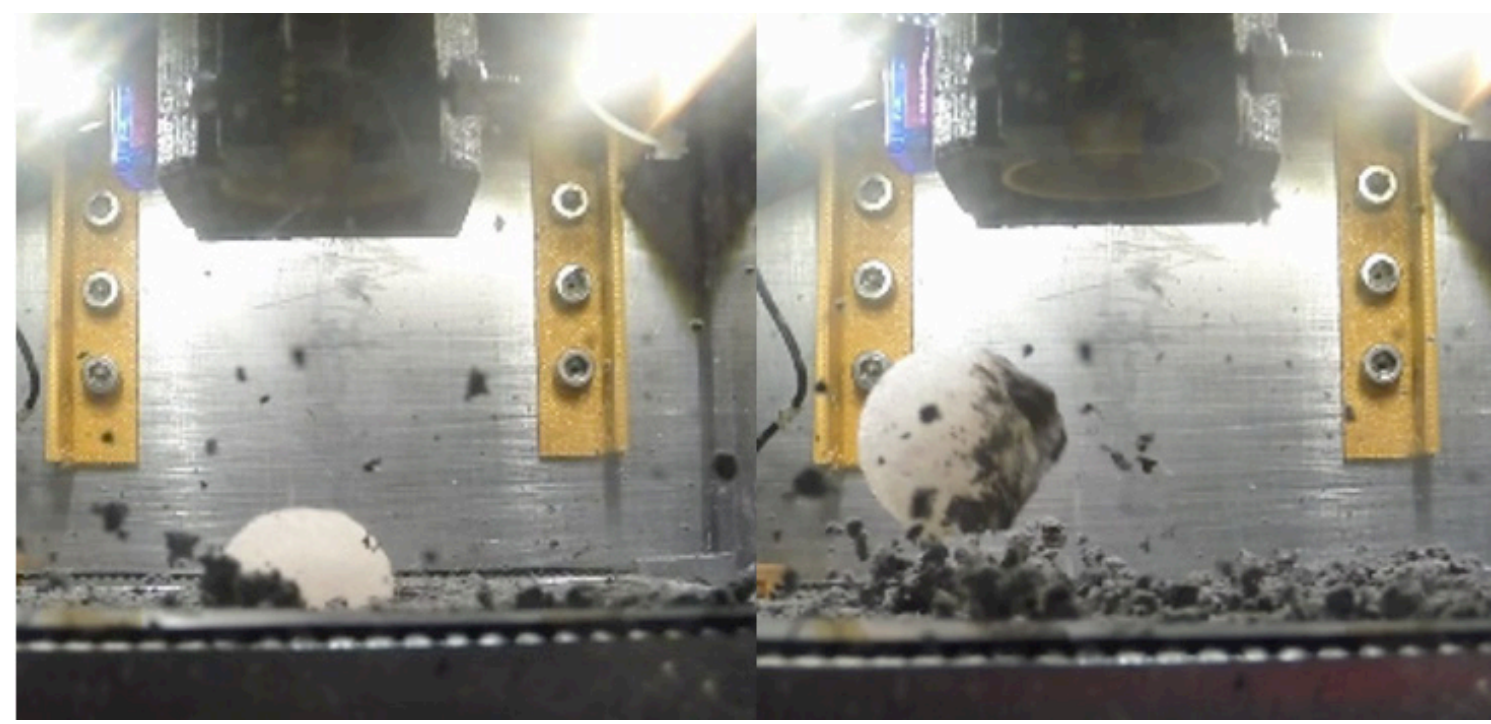

Figure 2. Still frame of 10-g quartz sand coated impactor (left) contacting Orgueil at an impact speed of $52 \mathrm{~cm} / \mathrm{s}$ and (right) rebounding with observable mass transfer.

COLLIDE-3 and PRIME-4, respectively. The target regolith used in the experiments described in this paper consisted of quartz sand, JSC-1 lunar regolith simulant (McKay et al., 1994), and $\mathrm{Cl}$ carbonaceous asteroid regolith simulant (hereafter referred to as Orgueil; Britt et al., 2019). Quartz sand was selected as a well-studied material with rounded grains for a more direct comparison to numerical simulations. JSC-1 and Orgueil regolith simulants were selected to study the effect of jagged grain types relevant for the exploration of airless bodies where grains evolve due to micrometeoroid bombardment as opposed to the aeolian processes that produce the rounded features of terrestrial quartz sand grains. The particle size distributions selected for these experiments were $75-250 \mu \mathrm{m}, 125-250 \mu \mathrm{m}$, and 250-500 $\mu \mathrm{m}$ to represent the distribution of fines anticipated on a variety of asteroids up to the size of the Moon. Thermal inertia measurements inferring regolith size distributions for 15 of 26 asteroids for which data were available to indicate particle size distributions $<1 \mathrm{~mm}$ (Gundlach \& Blum, 2013), consistent with our selected particle sizes.

To follow up on these observations, we developed an experimental apparatus to be used in a laboratory drop tower ( 0.75 second free-fall time) that simulates the rebound portion of these mass-transfer collision events without the time and cost requirements imposed by a flight-based experiment (Jarmak, 2020). The apparatus consists of a spring attached to a marble that is initially resting on a bed of regolith. When the apparatus is released from the top of the drop tower, the free-fall environment allows the spring to retract and pull the marble out of the regolith at accelerations between $1 \mathrm{~m} / \mathrm{s}^{2}$ and $9 \mathrm{~m} / \mathrm{s}^{2}$. We initially carried out our drop tower experiments in an open-air chamber before transitioning the apparatus into 
an evacuated chamber to provide a more direct comparison to the flight experiments.

In this paper, we provide the design and operation of our flight and ground-based experiments, explore the influence of projectile impact velocity and rebound acceleration on mass transfer events, identify whether a microgravity environment is needed to observe these mass transfer events, and assess the extent to which we can directly compare our flight and ground-based microgravity experiment results.

\section{MATERIALS AND METHODS}

\section{Parabolic Flight Experiments: PRIME}

The PRIME-3 and PRIME-4 parabolic flight experiments consisted of free-floating boxes each with a spring-loaded launcher containing a $\mathrm{cm}$-size spherical projectile above a tray of regolith (Figure 3 ). The target material is prepared by pouring the regolith into a rectangular tray and leveling the top of the granular bed with a ruler. The porosity is estimated to be between 0.4 and 0.5 based on laboratory measurements of the regolith weight contained in the tray (Brisset et al., 2018).

\section{PRIME-3}

The PRIME-3 parabolic flight campaign was carried out during four flights in August 2014. The experiment consisted of eight free-floating vacuum chamber boxes each housing a springloaded launcher containing a cm-size marble arranged over a tray of regolith that was initially covered by an aluminum door. The experiment parameters for the PRIME-3 flight campaign are provided in Table 1, and an example of a mass transfer event from PRIME-3 is shown in Figure 3. Thorough descriptions of the experiment hardware are found in Colwell et al. (2008) and Brisset et al. (2018).

\section{PRIME-4}

The PRIME-4 parabolic flight campaign was carried out during three flights, two in November 2017 and one in October 2018. The experiment consisted of 12 free-floating vacuum chamber boxes each housing a spring-loaded launcher containing a cm-size marble that was arranged over a tray of regolith covered by a cloth door. The experiment parameters for the PRIME-4 flight campaign are provided in Table 2, and an example of a mass transfer event from PRIME-4 is shown in Figure 2.

\section{Suborbital Flight Experiment: COLLIDE}

The COLLIDE-3 suborbital flight experiment was carried out in April 2016. The experiment consisted of four individual Impactor Box Systems (IBS) with three single launcher

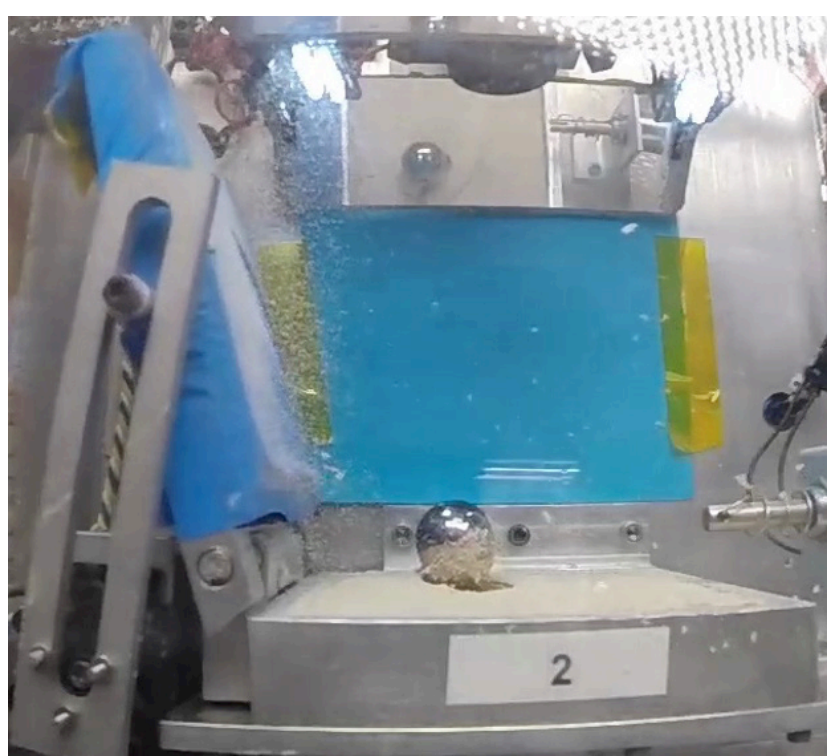

Figure 3. Example of a PRIME-3 experiment that resulted in the mass transfer of quartz sand onto a $31-\mathrm{g}$ steel marble.

Table 1. PRIME-3 experiment parameters.

\begin{tabular}{ccccc}
\hline & Projectile & & \multicolumn{2}{c}{ Regolith } \\
\hline $\begin{array}{c}\text { Diameter } \\
(\mathbf{c m})\end{array}$ & Mass $(\mathbf{g})$ & Material & Grain type & $\begin{array}{c}\text { Grain size } \\
(\boldsymbol{\mu m})\end{array}$ \\
\hline 1.9 & 10 & Quartz & JSC-1 & $125-250$ \\
& 31 & Steel & Quartz Sand & $75-250$ \\
\hline
\end{tabular}

Table 2. PRIME-4 experiment parameters.

\begin{tabular}{ccccc}
\hline & Projectile & & \multicolumn{2}{c}{ Regolith } \\
\hline $\begin{array}{c}\text { Diameter } \\
(\mathbf{c m})\end{array}$ & Mass & Material & Grain type & $\begin{array}{c}\text { Grain size } \\
(\boldsymbol{\mu m})\end{array}$ \\
\hline 1.9 & 10 & Quartz & JSC-1 & $125-250$ \\
& & & & $250-500$ \\
& & Sand & Orgueil & $125-250$ \\
& & Coated & & \\
& & Quartz & & \\
\hline
\end{tabular}

systems and one multi-launcher system (with three impactors). The single launcher systems were conceptually identical to the PRIME experiment systems and consisted of a spring-loaded launcher containing a cm-size marble arranged over a tray of regolith covered by an aluminum door. All experiments were operated under vacuum conditions. Details on the COLLIDE hardware can be found in Colwell (2003) and Brisset et al. (2018).

Only one of these four experiments resulted in an observable rebound of the launched marble; the parameters for the successful experiment are listed in Table 3. 
Table 3. COLLIDE-3 experiment parameters.

\begin{tabular}{ccccc}
\hline & Projectile & & \multicolumn{2}{c}{ Regolith } \\
\hline $\begin{array}{c}\text { Diameter } \\
(\mathbf{c m})\end{array}$ & Mass & Material & Grain type & $\begin{array}{c}\text { Grain size } \\
(\mu \mathrm{m})\end{array}$ \\
\hline 1.9 & 10 & Quartz & Quartz Sand & $<250$ \\
\hline
\end{tabular}

Table 4. Tabletop experiment parameters.

\begin{tabular}{ccccc}
\hline \multicolumn{2}{c}{ Spring pendulum parameters } & \multicolumn{2}{c}{ Regolith parameters } \\
\hline Mass $(\mathbf{g})$ & $\begin{array}{c}\text { Spring } \\
\text { constant } \\
(\mathbf{N} / \mathrm{m})\end{array}$ & $\begin{array}{c}\text { Spring } \\
\text { length }(\mathrm{m})\end{array}$ & Grain type & $\begin{array}{c}\text { Grain size } \\
(\boldsymbol{\mu m})\end{array}$ \\
\hline 31 & 3.5 & 0.27 & Quartz Sand & $75-250$ \\
& & & $200-500$ \\
& & JSC-1 & $<75$ \\
& & & $75-100$ \\
& & & $125-250$ \\
\hline
\end{tabular}

\section{Ground-Based Experiments}

We developed a tabletop experiment designed to produce low-velocity collisions of a $\mathrm{cm}$-size projectile into regolith at speeds under $1 \mathrm{~m} / \mathrm{s}$ to test whether the observed mass transfer phenomena from the flight experiments could be replicated under 1-g conditions. The apparatus for this experiment was composed of an adjustable, metal T-shaped stand supporting a spring attached to a spherical mass (Figure 4).

The experiment parameters are listed in Table 4. The projectile was approximately the same mass and diameter as the heaviest projectiles used in the PRIME-3 experiments, and the regolith material was selected to replicate the regolith used in the PRIME-3 campaign. Impact velocities were varied between $25 \mathrm{~cm} / \mathrm{s}$ and $60 \mathrm{~cm} / \mathrm{s}$.

\section{Drop Tower Experiment}

We modified the spring pendulum system used for our $1-\mathrm{g}$ experiments to make use of our laboratory's $3.7-\mathrm{m}$ drop tower which provides a microgravity environment for $\sim 0.75 \mathrm{~s}$ via freefall. This experimental apparatus was designed to simulate the rebound portion of the mass-transfer collision events observed in the flight experiments. The initial experiments were performed in ambient atmospheric conditions and the apparatus was later transitioned to an evacuated chamber.

\section{Open-air drop tower experiment}

Our open-air drop tower experiment apparatus (Figure 5) consisted of a tube containing a $\mathrm{cm}$-diameter marble suspended from a spring that begins in contact with a bed of regolith. When dropped in the drop tower the spring contracts during free-fall, thereby simulating the rebound portion of a low-velocity collision.

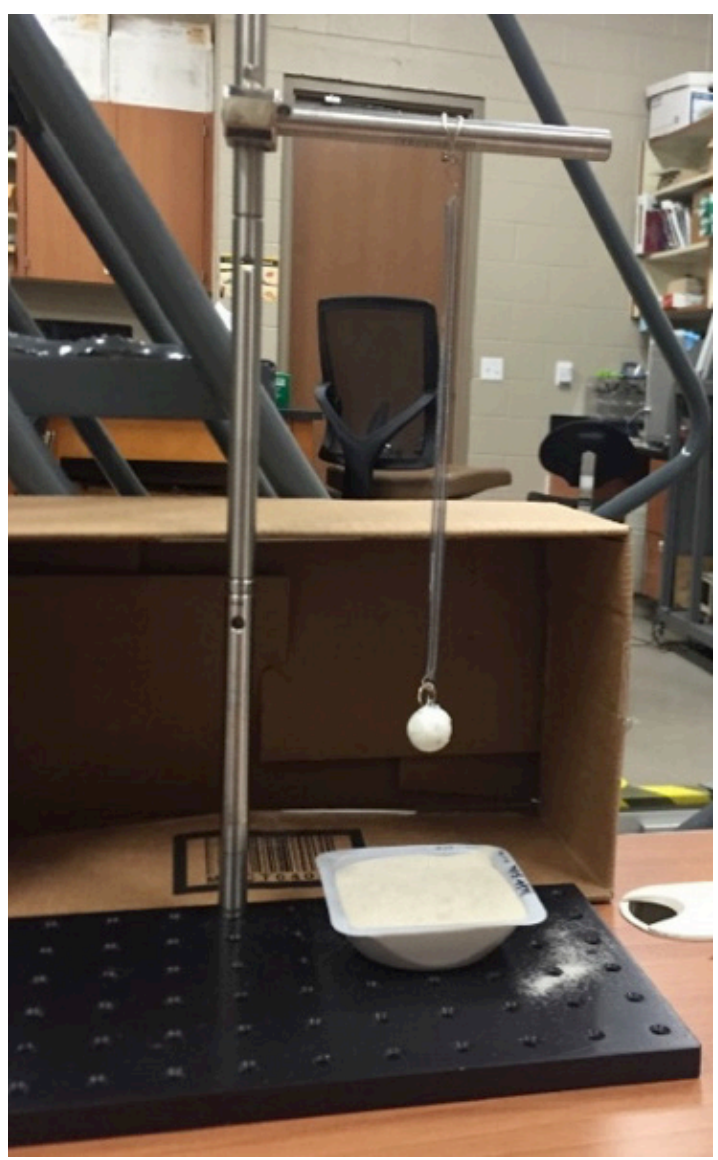

Figure 4. 1-g granular impact experiment spring pendulum apparatus.

We carried out a total of 47 microgravity rebound experiments with the open-air drop tower apparatus. The experiment parameters are listed in Table 5 and Figure 6 provides an example of a mass transfer event. The experiments were recorded by a GoPro Hero3+ camera at 240 or 120 fps with 420 or $720 p$ resolution, respectively; the experiments were initially recorded at $240 \mathrm{fps}$ with $420 \mathrm{p}$ resolution, but subsequent experiments were later carried out at $120 \mathrm{fps}$ due to the improved resolution achievable with the reduction in framerate. A framerate of $120 \mathrm{fps}$ would produce $\sim 94$ frames covering the $\sim 0.75 \mathrm{~s}$ free-fall duration which was sufficient to track the change in position of the projectile, and the improvement of the image resolution aided the detection of mass transfer events.

\section{Vacuum drop tower experiment}

Our preliminary drop tower experiment design was useful as a proof of concept to demonstrate that mass transfer events could be observed through low-energy interactions in a freefall environment in the laboratory. To produce data under 


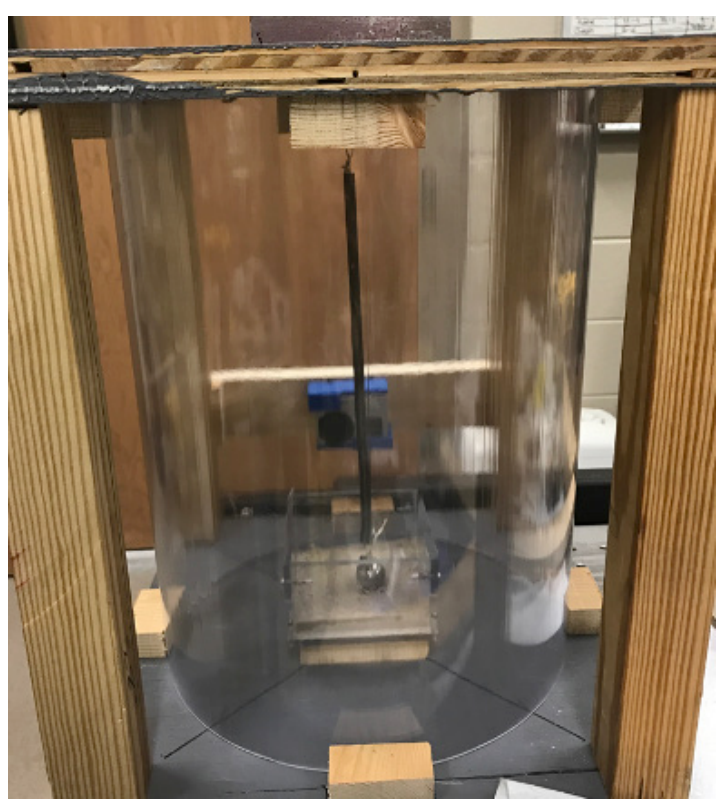

Figure 5. Open-air drop tower experiment apparatus.

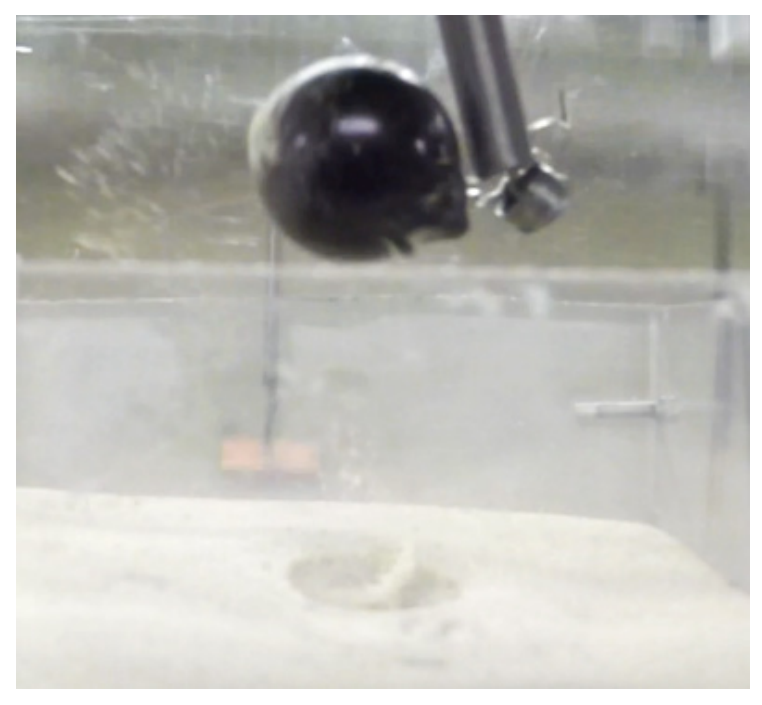

Figure 6. Still frame of 31-g steel projectile with observable mass transfer from quartz sand. Contrast enhanced for mass transfer visibility.

conditions relevant to airless bodies, and for a more direct comparison to the flight experiments, we transitioned the drop tower apparatus into a vacuum chamber (Figure 7).

The experiment apparatus internal to the vacuum chamber remained identical to the open-air apparatus and consisted of a projectile suspended from a spring resting in a bed of regolith. We carried out a total of 158 experiments with the vacuum drop tower apparatus. The experiment parameters for all cases are listed in Table 6.

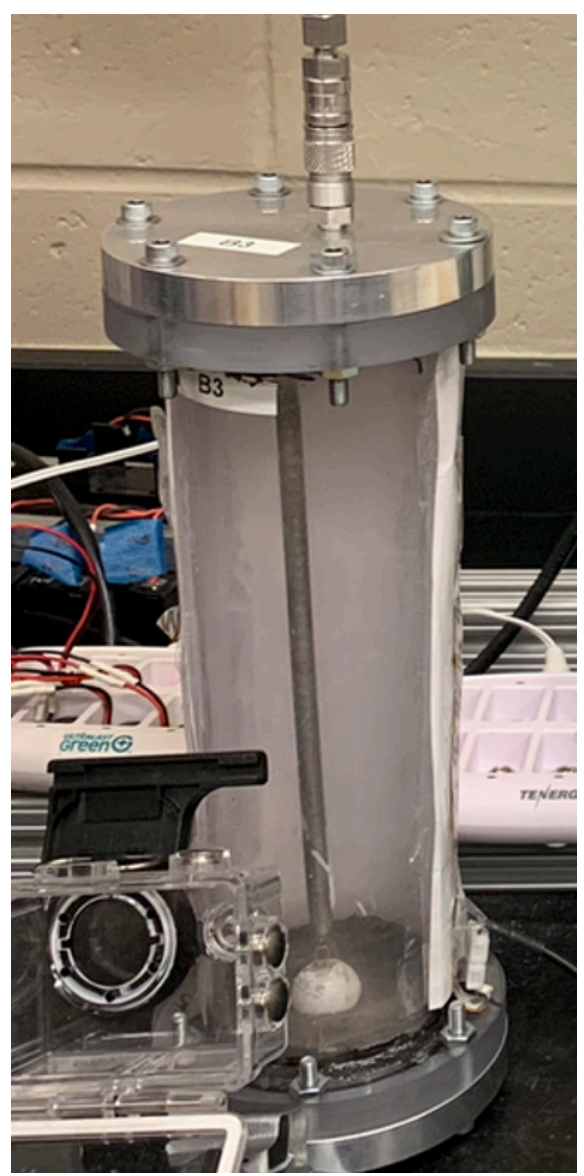

Figure 7. Vacuum drop tower apparatus. The chamber is detached from a vacuum pump shortly before an experiment is performed, and a Swagelok quick-disconnect fitting maintains the vacuum inside the tube for the duration of the experiment.

Table 5. Open-air drop tower experiment parameters.

\begin{tabular}{ccccc}
\hline & Projectile & & \multicolumn{2}{c}{ Regolith } \\
\hline $\begin{array}{c}\text { Diameter } \\
(\mathbf{c m})\end{array}$ & Mass $\mathbf{( g )}$ & Material & Grain type & $\begin{array}{c}\text { Grain size } \\
(\boldsymbol{\mu m})\end{array}$ \\
\hline 1.9 & 10 & Quartz & Quartz Sand & $75-250$ \\
& & & JSC-1 & $125-250$ \\
& \multirow{2}{*}{31} & Steel & & $250-500$ \\
& & & Orgueil & $125-250$ \\
\hline
\end{tabular}

\section{Measurements}

The measurable parameters considered in this paper include the projectile impact velocity (for the flight experiments only) and the projectile rebound acceleration. We only compare the rebound acceleration across the drop tower and flight data sets because the drop tower experiments do not allow for the impact of the projectile and the spring applies a force to the projectile. Therefore, we used acceleration to represent the motion of the projectile rather than assigning 
Table 6. Vacuum drop tower experiment parameters.

\begin{tabular}{ccccc}
\hline & Projectile & & \multicolumn{2}{c}{ Regolith } \\
\hline $\begin{array}{c}\text { Diameter } \\
(\mathbf{c m})\end{array}$ & Mass $(\mathbf{g})$ & Material & Grain type & $\begin{array}{c}\text { Grain size } \\
(\boldsymbol{\mu m})\end{array}$ \\
\hline 1.9 & 10 & Quartz & Quartz Sand & $\begin{array}{c}75-250 \\
250-500 \\
\end{array}$ \\
& 31 & Steel & JSC-1 & $125-250$ \\
2.54 & 20 & & & \\
& 67 & & & $250-500$ \\
3.81 & 226 & & Orgueil & $125-250$ \\
\hline
\end{tabular}

a constant velocity, and measuring impact velocities were no relevant for the drop tower data set because no impact occurred.

We used ImageJ (Schneider et al., 2012), a Javabased image processing program, to track the movement of the projectile in each experiment and perform our required measurements. The images are spaced apart in time based on the frame rate of the recording, which was either 120 or $240 \mathrm{fps}$. To maintain consistency in the results we tracked for $\sim 83 \mathrm{~ms}$ after the rebound of the projectile for the rebound acceleration measurement, which corresponds to 10 frames and 20 frames for $120 \mathrm{fps}$ and $240 \mathrm{fps}$ recordings respectively. These measurements were made for the open-air drop tower, vacuum drop tower, and flight experiments. For the impact velocity measurements in the flight data, we tracked the projectile as soon as it was visible from the launcher until it made contact with the bed of regolith.

For each experiment, we qualitatively assigned the mass transfer outcomes (hereafter MTOs) as either none, low, medium, or high. We made several attempts to define a repeatable quantitative measure of the amount of transferred mass, such as measuring the area of the grains adhered to the projectile surface or estimating a cone of material and an assumed regolith density. However, variable image quality and geometry differences in the observations, as well as potentially large variations in the density of the adhered material due to variable compaction, made it impossible to have reliable quantitative results with more precision than our 4-level designation of "none", "low", "medium", and "high". Therefore, we classified the qualitative MTOs based on the following criteria (examples shown in Figure 8):

- None: No visible mass transfer at all, or only a monolayer of material consistent with our 1-g experiment observations.

- Low: Clearly visible layer more significant than a monolayer, but minimal thickness beyond the projectile surface.

- Medium: Clearly visible extent of regolith beyond the surface of the projectile, potentially in a strip or conical shape.
- High: The projectile has a significant cone of regolith adhered to its surface or a clearly identifiable thick layer covering a large portion of the projectile surface.

\section{RESULTS}

\section{Flight Experiments}

Of the 19 COLLIDE and PRIME experiments that resulted in the rebound of the projectile from the bed of regolith, 14 resulted in observable mass transfer. Figure 9 shows examples of observations yielding mass transfer from the flight experiments, which resulted in more significant MTOs than observed in any of the drop tower experiments. The experiment parameters and measurements corresponding to those mass transfer events are provided in Table 7.

Figure 10 shows the relationship between MTO and projectile impact velocity for the flight data. We do not observe a clear dependence of MTO on impact velocity in the available data.

Figure 11 shows the relationship between MTO and projectile rebound acceleration for the flight data which indicates that MTO outcomes of 'medium' and 'high' only occur for rebound accelerations $<0.09 \mathrm{~m} / \mathrm{s}^{2}$ and $0.38 \mathrm{~m} / \mathrm{s}^{2}$, respectively. MTOs occurred for rebound accelerations as high as $5.4 \mathrm{~m} / \mathrm{s}^{2}$, and no mass transfer occurred for rebound accelerations as low as $0.029 \mathrm{~m} / \mathrm{s}^{2}$. Nearly all of the flight data had rebound acceleration measurements $<2 \mathrm{~m} / \mathrm{s}^{2}$ with one outlier measurement at $5.4 \mathrm{~m} / \mathrm{s}^{2}$. It would be beneficial to obtain flight data with rebound accelerations between $2 \mathrm{~m} /$ $\mathrm{s}^{2}$ and $5 \mathrm{~m} / \mathrm{s}^{2}$ as well as accelerations $>5 \mathrm{~m} / \mathrm{s}^{2}$ for additional overlap with the drop tower data sets and further insight into the acceleration thresholds influencing mass transfer. We found that a more significant fraction of the flight experiments yielded "medium" and "high" mass transfer results compared to the drop tower experiments which were unable to replicate "high" mass transfer results at all.

\section{1-g Experiments}

Each impact produced only a monolayer of granular material onto the projectile. This result is inconsistent with the observations produced by PRIME and COLLIDE where a more significant mass transfer is observed. Additionally, simply pressing the impactor into the regolith [thus dramatically increasing the contact forces and therefore the cohesive strength of the material (Sanchez \& Scheeres, 2014)] and pulling it up produced identical results to the spring pendulum impacts, i.e. no significant transfer of material. The median grain value of our samples was $100 \mu \mathrm{m}$ so we would anticipate cohesive forces near $10^{-15} \mathrm{~N}$ and gravitational forces near $10^{-8} \mathrm{~N}$ (Brisset et al., 2018). We concluded that the cohesion force between the grains was insufficient to overcome the 


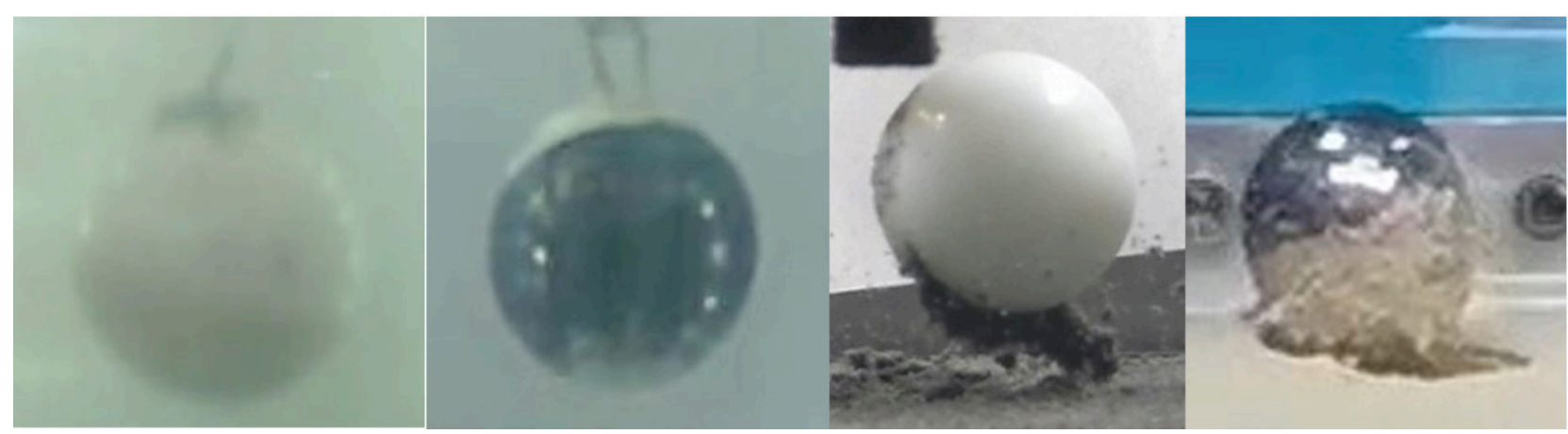

Figure 8. From left to right: Example of no mass transfer (monolayer), low mass transfer, medium mass transfer, and high mass transfer.
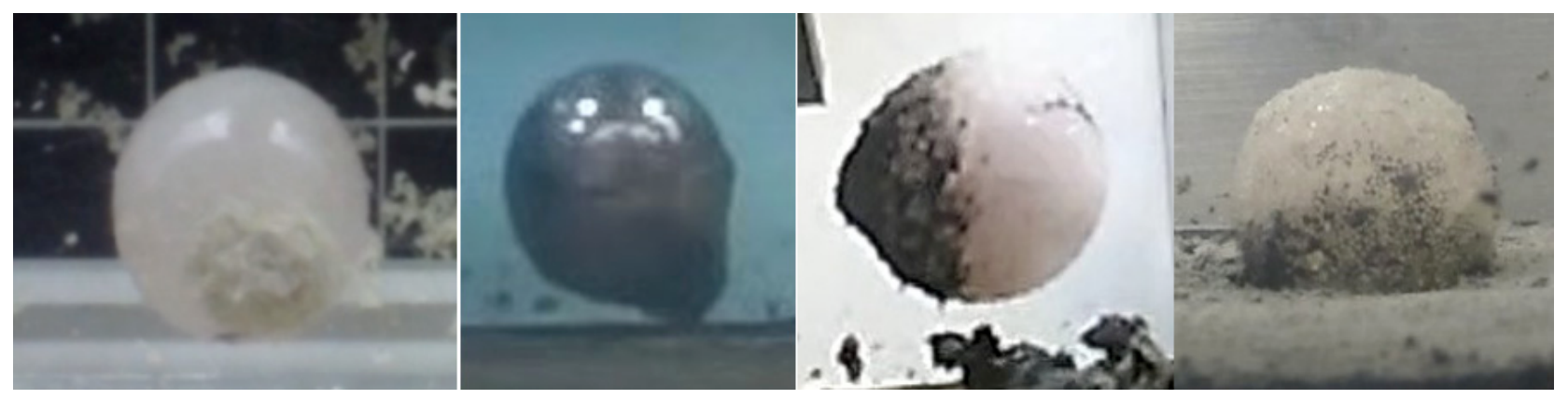

Figure 9. Observations of mass transfer from regolith onto $\mathrm{cm}$-size marbles in various flight experiments.

Table 7. Experiment parameters yielding mass transfer for the flight experiments.

\begin{tabular}{|c|c|c|c|c|}
\hline Regolith $(\mu \mathrm{m})$ & Marble mass & Impact velocity (cm/s) & Rebound acceleration $\left(\mathrm{m} / \mathrm{s}^{2}\right)$ & Mass transfer \\
\hline Orgueil (125-250) & 10 & 16.2 & 0.16 & Low \\
\hline JSC (125-250) & 10 & 27.8 & 0.31 & Low \\
\hline Orgueil (125-250) & 10 & 34.8 & 0.96 & Low \\
\hline Orgueil (125-250) & 10 & 51.8 & 5.40 & Low \\
\hline Quartz (75-250) & 10 & 23.2 & 0.004 & Medium \\
\hline JSC (125-250) & 31 & 29.6 & 0.07 & Medium \\
\hline JSC (125-250) & 31 & 14.9 & 0.08 & Medium \\
\hline JSC (125-250) & 31 & 4.1 & 0.09 & Medium \\
\hline JSC (125-250) & 10 & 4.1 & 0.09 & Medium \\
\hline Quartz (75-250) & 10 & 37.1 & 0.03 & High \\
\hline Quartz (75-250) & 10 & 38.5 & 0.03 & High \\
\hline Orgueil (125-250) & 10 & 31.8 & 0.11 & High \\
\hline Orgueil (125-250) & 10 & 26.6 & 0.16 & High \\
\hline Quartz (75-250) & 31 & 14.1 & 0.38 & High \\
\hline
\end{tabular}




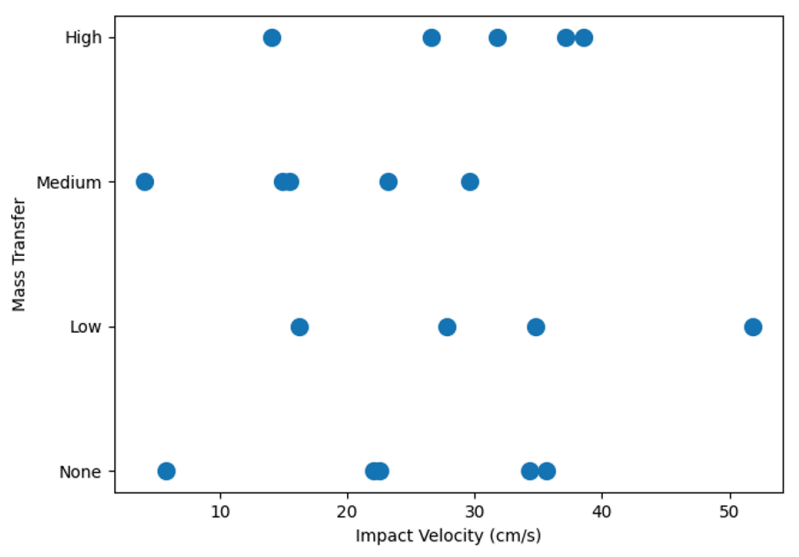

Figure 10. Mass transfer outcome vs. projectile impact velocity for flight experiments.

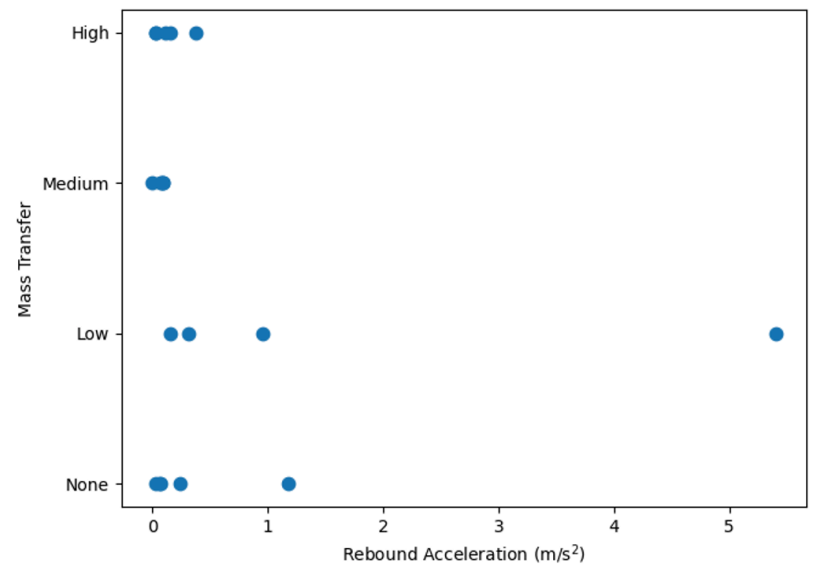

Figure 11. Mass transfer outcome vs. rebound acceleration for flight experiments.

force of gravity. Therefore, significant mass transfer due to a low-velocity collision between a $\mathrm{cm}$-size projectile and $\mathrm{mm}$ size grains requires a microgravity environment.

\section{Drop Tower Experiments}

We carried out a total of 205 drop tower experiments: 47 in open-air and 158 under vacuum conditions. We provide the observations from these drop tower experiments in the sections below.

\section{Open-air drop tower experiment}

We collected data from 47 open-air drop tower experiments, 16 of which resulted in the observable mass transfer of the target regolith simulant onto the projectile. Figure 12 shows examples of observations yielding mass transfer. From these images, we can see that less mass is transferred than
Table 8. Experiment parameters for experiments yielding mass transfer (MTO = "low") for open-air drop tower experiments.

\begin{tabular}{ccc}
\hline Regolith $(\boldsymbol{\mu m})$ & Marble mass & Rebound acceleration $\left(\mathbf{m} / \mathbf{s}^{2}\right)$ \\
\hline Quartz (75-250) & 31 & 3.7 \\
Quartz (75-250) & 31 & 4.5 \\
JSC (250-500) & 31 & 4.9 \\
JSC (250-500) & 31 & 5.1 \\
Quartz (75-250) & 31 & 5.5 \\
JSC (250-500) & 31 & 5.5 \\
JSC (250-500) & 31 & 5.6 \\
Quartz (75-250) & 31 & 5.8 \\
Quartz (75-250) & 31 & 5.8 \\
Orgueil (125-250) & 31 & 6.5 \\
JSC (250-500) & 31 & 6.6 \\
JSC (250-500) & 31 & 6.8 \\
JSC (250-500) & 31 & 7.4 \\
JSC (125-250) & 31 & 7.6 \\
JSC (125-250) & 31 & 7.7 \\
Quartz (75-250) & 31 & 7.8 \\
\hline
\end{tabular}

observed in the COLLIDE and PRIME experiments, but the quantities are more significant than a monolayer of granular material, thus we only have MTOs of "none" and "low" for this set of experiments. The experiment parameters and measurements corresponding to those mass transfer events are provided in Table 8.

While little mass transfer occurred, there are perhaps two bounds that can be set on MTOs for these experiments. First, no mass transfer was observed for rebound accelerations $>7.8 \mathrm{~m} / \mathrm{s}^{2}$. Second, no mass transfer was observed for marble masses $<31 \mathrm{~g}$. Rebound accelerations as low as $5.4 \mathrm{~m} / \mathrm{s}^{2}$ were achieved with marble masses of $10 \mathrm{~g}$, but the rebound accelerations, in general, were systematically higher (as expected given the spring pendulum nature of the system).

\section{Vacuum drop tower experiment}

To collect data at pressures more comparable to flight experiment conditions and more relevant to planetary environments of interest, we transferred the experiment apparatus to a vacuum chamber capable of achieving pressures down to $\sim 200$ mTorr. We carried out 158 drop tower experiments in low-pressure conditions, 33 of which resulted in observable mass transfer. Figure 13 shows examples of observations yielding mass transfer from these experiments. From these images, we see that less mass is transferred than observed in the COLLIDE and PRIME experiments, but there is more significant mass transferred than a monolayer 


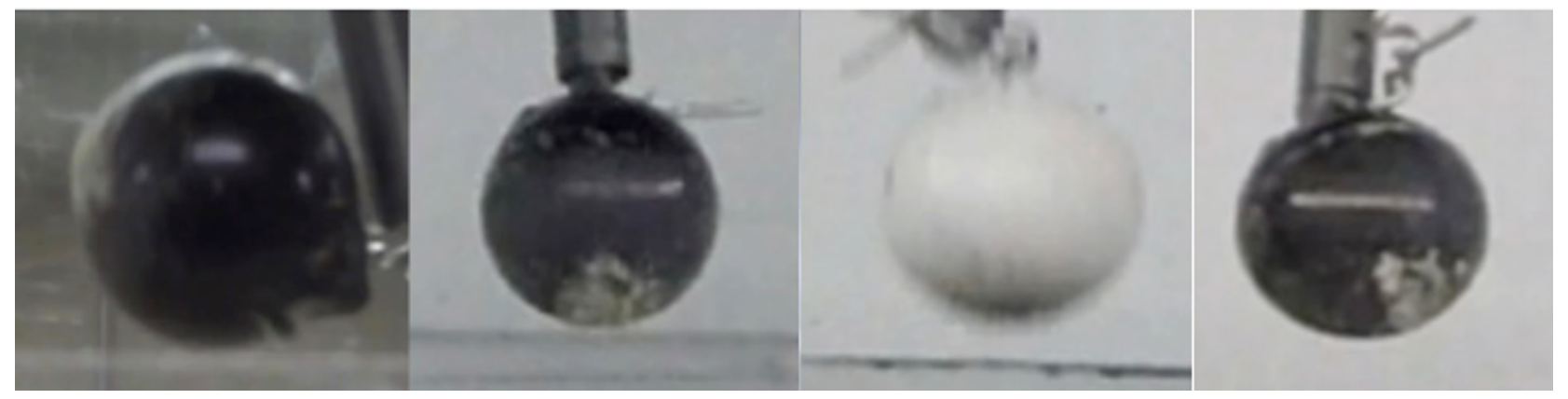

Figure 12. Observations of mass transfer from regolith onto $\mathrm{cm}$-size marble in an open-air, free-fall environment.

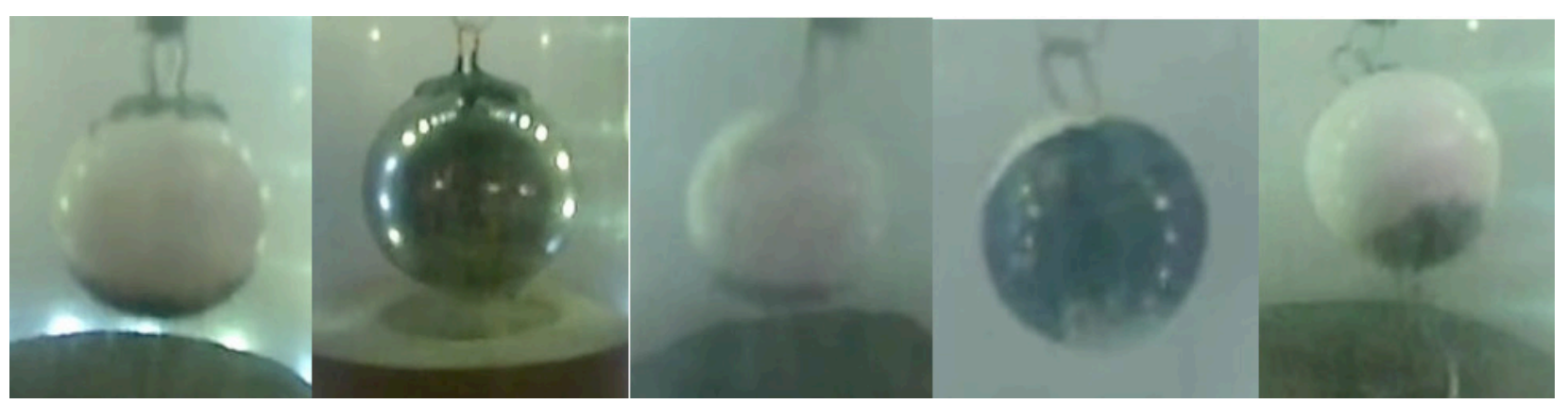

Figure 13. Observations of mass transfer from regolith onto $\mathrm{cm}$-size marble in a free-fall, vacuum environment.

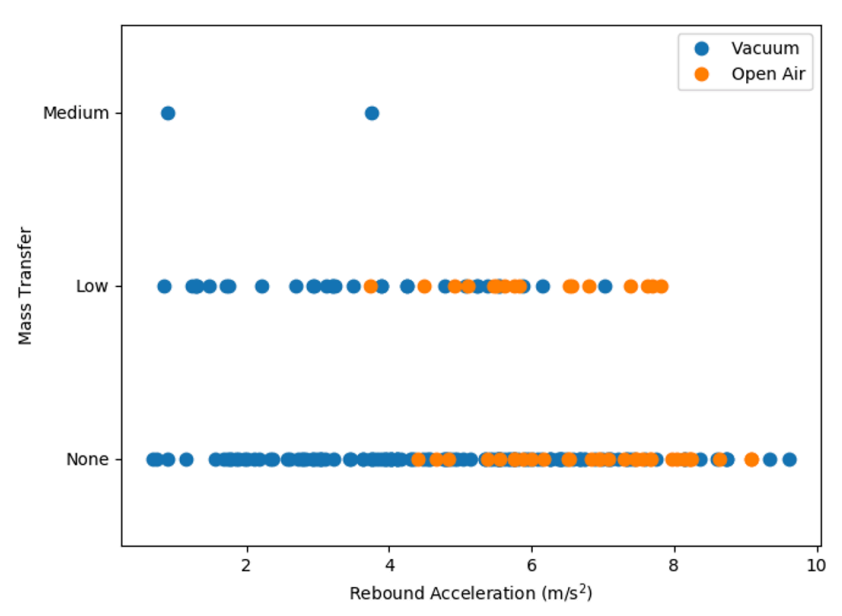

Figure 14. Mass transfer outcome vs. rebound acceleration for our open-air and vacuum drop tower experiments.

of granular material, with a few resulting in a "medium" MTO classification. The experiment properties corresponding to those mass transfer events are provided in Table 9.

Again, there appears to be a dependence of the MTO on rebound acceleration. Two experiments resulted in medium MTOs and these events occurred for rebound accelerations
$<3.8 \mathrm{~m} / \mathrm{s}^{2}$. A greater number of low MTOs were observed, all with rebound accelerations $<7.8 \mathrm{~m} / \mathrm{s}^{2}$ and MTOs occurred with marble masses as low as $10 \mathrm{~g}$. Finally, the resulting MTOs for both the open-air and vacuum drop tower experiments are shown in Figure 14, which shows that the parameter space explored by the vacuum drop tower experiment expands upon the open-air experiments to include lower rebound accelerations. Finally, comparing data from both the flight and open-air and vacuum drop tower experiments (Figure 15) suggests that more significant mass transfer is more likely for lower rebound accelerations, potentially explaining the higher percentage of MTOs in the flight data which predominantly occurred at lower values.

\section{DISCUSSION}

The goals of our experimental campaigns were (1) to identify projectile energy thresholds that influence MTOs in lowenergy collision events between $\mathrm{cm}$-size projectiles and $\mathrm{mm}$ size regolith, (2) to determine whether these MTOs required a microgravity environment to be observed, and (3) to determine whether the rebound portion of these collision events could be replicated in a laboratory drop tower environment. In the 
Table 9. Experiment parameters yielding mass transfer for our vacuum drop tower experiments.

\begin{tabular}{|c|c|c|c|}
\hline Regolith $(\mu \mathrm{m})$ & $\begin{array}{c}\text { Marble } \\
\text { mass }\end{array}$ & $\begin{array}{c}\text { Rebound } \\
\text { acceleration }\left(\mathrm{m} / \mathrm{s}^{2}\right)\end{array}$ & МтO \\
\hline JSC (250-500) & 67 & 0.89 & Medium \\
\hline JSC (250-500) & 67 & 3.8 & Medium \\
\hline Orgueil (125-250) & 226 & 0.84 & Low \\
\hline JSC (125-250) & 226 & 1.2 & Low \\
\hline JSC (125-250) & 226 & 1.3 & Low \\
\hline Orgueil (125-250) & 226 & 1.3 & Low \\
\hline Orgueil (125-250) & 226 & 1.3 & Low \\
\hline Quartz (250-500) & 67 & 1.5 & Low \\
\hline JSC (125-250) & 226 & 1.7 & Low \\
\hline JSC (125-250) & 226 & 1.8 & Low \\
\hline JSC (125-250) & 226 & 2.2 & Low \\
\hline Quartz (250-500) & 67 & 2.7 & Low \\
\hline Quartz (250-500) & 67 & 2.9 & Low \\
\hline Quartz (75-250) & 31 & 3.0 & Low \\
\hline Quartz (75-250) & 31 & 3.1 & Low \\
\hline Orgueil (125-250) & 67 & 3.2 & Low \\
\hline Orgueil (125-250) & 67 & 3.2 & Low \\
\hline Quartz (75-250) & 31 & 3.2 & Low \\
\hline Orgueil (125-250) & 67 & 3.5 & Low \\
\hline JSC (250-500) & 67 & 3.8 & Low \\
\hline Orgueil (125-250) & 67 & 3.9 & Low \\
\hline Quartz (75-250) & 10 & 4.3 & Low \\
\hline JSC (250-500) & 31 & 4.3 & Low \\
\hline Quartz (75-250) & 31 & 4.8 & Low \\
\hline Quartz (75-250) & 31 & 5.1 & Low \\
\hline Orgueil (125-250) & 31 & 5.2 & Low \\
\hline JSC (125-250) & 31 & 5.3 & Low \\
\hline Orgueil (125-250) & 31 & 5.4 & Low \\
\hline Quartz (75-250) & 31 & 5.5 & Low \\
\hline Quartz (75-250) & 10 & 5.6 & Low \\
\hline Orgueil (125-250) & 31 & 5.9 & Low \\
\hline Quartz (75-250) & 10 & 6.2 & Low \\
\hline Orgueil (125-250) & 31 & 7.0 & Low \\
\hline
\end{tabular}

following paragraphs, we discuss both the successes and limitations of our experimental campaigns in achieving each of these goals along with the implications of our results.

Our data suggest that projectile rebound acceleration influences the resulting MTOs, with lower rebound accelerations leading to higher MTOs. We did not, however, identify a relationship between impact velocity and MTO from our available flight data. The impact velocity directly influences the rebound acceleration of the projectile and so if a relationship between projectile rebound acceleration and MTO exists we would expect the same to be true for the projectile impact velocity. However, the rebound acceleration is also a function of the material properties of the granular bed, so the lack of a clear relationship between impact velocity and MTO may be due to both the limited available data (impact velocities were only available for flight experiments) and variations of the granular bed material properties in each experiment obfuscating the relationship. Therefore, additional experiments varying the projectile impact velocity at higher granularity for various regolith compositions and particle size distributions are needed to quantify the relationship between projectile impact velocity and MTO.

We found that mass transfer events occurred for rebound accelerations $<7.8 \mathrm{~m} / \mathrm{s}^{2}$, and MTOs of "medium" and "high" occurred for rebound accelerations $<3.8 \mathrm{~m} / \mathrm{s}^{2}$ and $0.38 \mathrm{~m} / \mathrm{s}^{2}$ respectively when considering the drop tower and flight data combined. When considering the flight data alone, however, the rebound acceleration threshold for "medium" MTOs is significantly lower than in the combined data set with a value of $0.09 \mathrm{~m} / \mathrm{s}^{2}$. However, this could be due to our limited quantity of available flight data. There is a possibility to produce medium MTOs through in-flight experiments with projectile rebound accelerations within a higher threshold, but we did not capture these events because we were limited to 25 impact experiments composed of a variety of explored parameters each of which may influence MTOs in addition to the rebound acceleration and therefore a more systematic study is needed. Additionally, particles with insufficient cohesion to maintain contact throughout the pull-off force would be left behind in the experiments with higher rebound accelerations leading to an overall lower number of grain contacts and thus lower observed mass transfer. It is also worth noting that triboelectric charging through grain interactions could play a role in the cohesive response of the material, but assessing the charged nature of the particles was beyond the scope of our experiments. Future investigations would benefit from evaluating the charged environment as an additional relevant variable influencing MTOs. Regardless, these rebound acceleration thresholds to produce MTOs are useful for the design of future experiments that intend to further investigate how various parameters influence MTOs and can still be used as inputs for numerical models characterizing the response of regolith to impacts in microgravity.

Additionally, given that several parameters were varied in each of our experiments, it may be misleading to consider only a single parameter at a time with our data set when investigating the thresholds that determine MTOs. For example, Figure 15 shows the MTO vs. rebound acceleration separated by flight and drop tower campaigns, but rebound acceleration was 


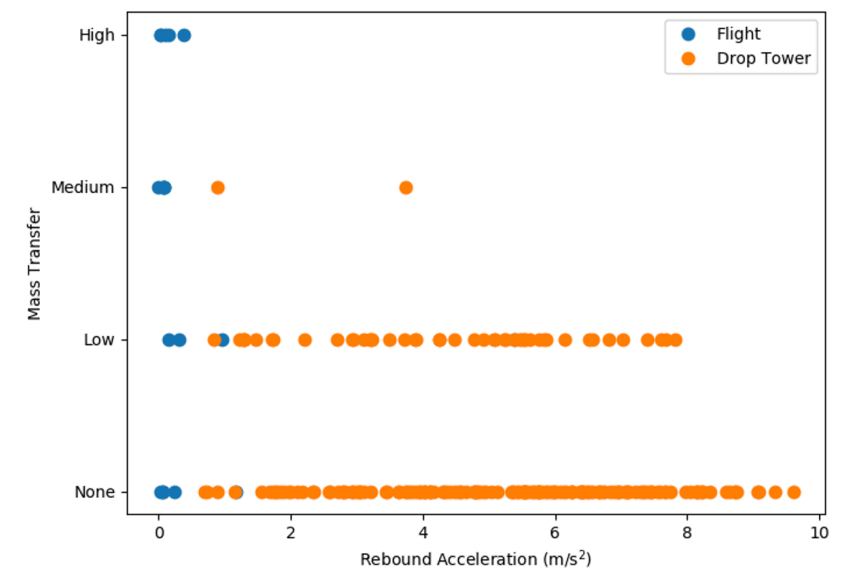

Figure 15. Mass transfer outcome vs. rebound acceleration for flight and drop tower data.

not the only parameter varied in each of these experiments. The regolith type and grain size distribution, projectile mass and diameter, extent to which the projectile was embedded in the regolith, and experiment pressure all served as separate sources of variability. Therefore, a more rigorous approach whereby the influence of each parameter on the MTO is statistically assessed through general factorial analysis, a statistical method that allows for a quantitative assessment of the relative level of influence of each considered parameter, would be an appropriate next step in the interpretation of this data. To carry out this statistical analysis the experiments would need to be balanced to each parameter of interest (Jarmak, 2020). We, therefore, recommend expanding the experimental campaign with parameter values selected through a factorial analysis experimental design to better account for the way each varied parameter and interaction effects between these parameters influence MTOs.

This full factorial investigation requires hundreds of experiments to fully explore the influence and interaction effects of the possible parameters involved (projectile mass, projectile diameter, projectile rebound acceleration, regolith material type, regolith grain size distribution, experiment pressure, etc.) to just two to three levels of granularity (e.g., assigning "low", "medium", and "high" bins to quantitative parameters including the rebound acceleration and projectile mass or groups such as "rounded" and "irregular" grain types for regolith). The number of required experiments to fully explore the parameter space is $n^{k}$ where $n$ is the level of desired granularity ( 2 for "low" and "high", 3 for "low", "medium", "high", etc.) and $\mathrm{k}$ is the number of parameters (Jarmak, 2020). For example, to investigate the influence of projectile mass, projectile diameter, projectile rebound acceleration, regolith grain type, regolith grain size, and experiment pressure and interactions between these parameters to two levels of granularity would require 64 experiments ideally with 3 replicates requiring 192 experiments. Given the long lead times associated with flight experiments and the limited number of possible experiments that can be performed during each flight, a campaign requiring over 200 experiments would cost thousands of dollars and take years to complete. A ground-based microgravity campaign via a laboratory drop tower is therefore ideal to broadly explore the influence of many parameters on MTOs, but careful consideration must be taken to calibrate the observations such that they can be directly compared to flight experiment results.

To determine whether mass transfer events observed in microgravity could still be reproduced in a terrestrial environment, we carried out a series of low-velocity impact experiments at 1-g under ambient atmospheric conditions. In each of our experiments, we observed that only a monolayer of grains adhered to the surface, inconsistent with the observed clumps of regolith that adhered to the surface of the projectile in our flight experiments. Therefore, we concluded that the force of gravity overcame the cohesive forces between the grains and therefore observations of significant MTOs require a much weaker gravity environment.

We were unable to replicate the 'high' MTOs from our flight campaigns with the drop tower campaign, and the observed MTOs were lower in the drop tower experiments than the flight experiments. The lack of 'high' MTOs in the drop tower campaign may be a direct result of the apparatus' inability to achieve sufficiently low rebound accelerations due to the dependence of the rebound acceleration on the spring constant of the selected spring and the projectile mass. Additionally, pulling a projectile gently away from the regolith surface may not sufficiently simulate the physics involved in the rebound of a projectile subsequent to an impact. For example, the impact of the projectile alters the compaction of the grains at the site of impact in a collision whereas in our drop tower experiments the grains are compacted due to the weight of the projectile at rest. However, time limitations in drop towers restrict our ability to directly replicate a full collision event, and the flight and drop tower data sets may not be directly comparable. Numerical investigations into these distinct physical phenomena could perhaps reveal whether a quantifiable relationship between the two exists. If such a relationship exists, we could develop a scaling law that would allow us to transform the drop tower data MTO results into the MTO of a corresponding impact event.

\section{CONCLUSION}

We collected data investigating low-energy disturbances of regolith under $1-\mathrm{g}$ and microgravity conditions through three flight-based, one ground-based, and two drop tower 
experiment campaigns. The flight campaigns (PRIME-3, PRIME-4, and COLLIDE) took place on a parabolic airplane and suborbital flights allowing sufficient time to observe the impact and rebound of a $\mathrm{cm}$-size projectile into regolith at speeds of $10 \mathrm{~s}$ of $\mathrm{cm} / \mathrm{s}$. The $1 \mathrm{gg}$ experiments included lowvelocity $(<60 \mathrm{~cm} / \mathrm{s})$ impacts of a $\mathrm{cm}$-size projectile into regolith under 1-g conditions. The drop tower campaigns were limited by a free-fall time of $\sim 0.75 \mathrm{~s}$ and so only the rebound portion of an impact event could be simulated and subsequently observed for the desired low-energy regime.

In our flight and drop tower experiments we found that, under certain conditions, a mass transfer event occurs where regolith from the target granular bed adheres to the surface of the projectile disturbing the bed. We investigated the dependence of MTOs on rebound acceleration of the projectile and found that mass transfer events occurred for rebound accelerations $<7.8 \mathrm{~m} / \mathrm{s}^{2}$, and future experiments investigating various parameter thresholds that govern the occurrence of mass transfer should aim for projectile rebound accelerations below this value to increase the likelihood of observing mass transfer events.

Our 1-g impact experiments resulted in only a monolayer of granular material adhering to the surface of the projectile, suggesting that the grains were not cohesive enough to overcome the force of gravity. These results indicated that significant mass transfer due to a low-velocity collision between a $\mathrm{cm}$-size projectile and $\mu \mathrm{m}$-size grains requires a microgravity environment. Additionally, our available data from the microgravity experiment campaigns did not reveal a relationship between impact velocity and MTOs, but we found that lower rebound accelerations of projectiles tend to produce more significant MTOs. However, given the varied nature of the data (differences in regolith grain size and composition, projectile mass and diameter, experiment pressure environment, etc.) it may be misleading to consider the influence of a single parameter at a time on MTOs. Therefore, a thorough study separating the effect of each varied parameter and their interactions is needed to better ascertain the parameter thresholds that govern the occurrence of mass transfer events in microgravity. We also found that transfer outcomes for a projectile impacting and rebounding from a granular bed and a projectile pulled from a granular bed result have different results: the pulled projectile MTOs are consistently lower than the projectile that rebounds after impact. Therefore, the MTOs may not be directly comparable and a scaling law applied to the drop tower data results may be necessary to combine the data sets.

Given that our drop tower experimental results may not capture the full physics of the rebound portion of a collision event, flight experiments remain our high-fidelity option for investigating the mechanical response of regolith to lowenergy disturbances in microgravity. However, the time required to acquire the hundreds of experiments to fully investigate the influence of parameters and interactions between those parameters on MTOs is prohibitive. Drop tower campaigns therefore remain the best option for broadly exploring the available parameter space, but the data must be considered in tandem with comparable flight data to appropriately interpret the results. Therefore, increasing the number of available flight and drop tower experiments investigating low-energy disturbances of regolith in microgravity through a systematic factorial experiment design plan would expand our understanding of the parameters that influence MTOs thereby improving our understanding of the surfaces of small planetary bodies.

\section{REFERENCES}

Gundlach B,Blum J (2013) A new method to determine the grain size of planetary regolith. Icarus 223, 479-492.

Binzel RP (2014) Human spaceflight: find asteroids to get to Mars. Nature News 514, 559.

Brisset J, Colwell J, Dove A, Abukhalil S, Cox C, Mohammed N (2018) Regolith behavior under asteroid-level gravity conditions: low-velocity impact experiments. Progress in Earth and Planetary Science 5, 1-21.

Britt DT, Cannon KM, Donaldson Hanna K, Hogancamp J, Poch O (2019) Simulated asteroid materials based on carbonaceous chondrite mineralogies. Meteoritics \& Planetary Science 54, 2067-2082.

Colwell JE (2003) Low velocity impacts into dust: results from the COLLIDE-2 microgravity experiment. Icarus 164, 188-196.

Colwell JE, Sture S, Cintala M, Durda D, Hendrix A, Goudie T, Route $\mathrm{M}(2008)$ Ejecta from impacts at $0.2-2.3 \mathrm{~m} / \mathrm{s}$ in low gravity. Icarus $195,908-917$.

Jarmak S (2020) Experimental and Numerical Investigations of Granular Dynamics in Microgravity. Orlando: University of Central Florida.

Lauretta DS, DellaGiustina DN, Bennett CA, Golish DR, Becker KJ, Balram-Knutson SS, Hamilton V (2019) The unexpected surface of asteroid (101955) Bennu. Nature 568, 55-60.

McKay DS, Carter JL, Boles WW, Allen CC, Allton JH (1994) JSC1: a new lunar soil simulant. Engineering, Construction, and Operations in Space IV 2, 857-866.

Sanchez P, Scheeres DJ (2014) The strength of regolith and rubble pile asteroids. Meteoritics \& Planetary Sciences 49, 788-811.

Schneider CA, Rasband WS, Eliceiri KW (2012) NIH Image to ImageJ: 25 years of image analysis. Nature methods 9, 671-675.

Smith K (2019) Hayabusa2 at the asteroid Ryugu. Science 364, 249-250.

Evolution in Protoplanetary Disks. Protostars and Planets, 914. 